\title{
Early chemosensitivity of normal hematopoietic cells and malignant lymphoblasts predicts relapse in childhood acute lymphoblastic leukemia
}

\author{
TAMER H. HASSAN \\ Pediatric Hematology and Oncology Unit, Zagazig University, Zagazig, Egypt
}

Received August 27, 2010; Accepted November 1, 2010

DOI: $10.3892 / \mathrm{ol} .2010 .203$

\begin{abstract}
For the last 30 years, numerous clinical and biological pretreatment risk factors have been utilized for riskbased treatment assignment in childhood acute lymphoblastic leukemia (ALL). However, with improved chemotherapy regimens, many of these traditional prognostic factors have lost clinical significance. We aimed to improve relapse prediction in children with ALL through evaluation of the early chemosensitivity of normal and malignant cells and to determine the relationship between such chemosensitivity and risk of relapse. We retrospectively analyzed a cohort of 60 children with newly diagnosed ALL of whom 40 patients were in complete remission for at least 4 years and 20 patients relapsed during or following treatment. Time to peripheral blood blast clearance (PBBC) was used as a measure of chemosensitivity of malignant lymphoblasts while end-ofinduction complete blood count (CBC) parameters were used as a measure of chemosensitivity of normal hematopoietic cells. Our results showed that longer time to PBBC and lower end-of-induction total leukocyte count (TLC) and absolute neutrophil count (ANC) were significantly associated with increased risk of relapse. In conclusion, time to PBBC and end-of-induction TLC and ANC are important predictors of relapse and should be used to modify the intensity of chemotherapy at earlier time points during the course of treatment. A wider prospective, randomized, controlled trial is required to confirm our results.
\end{abstract}

\section{Introduction}

Although the majority of children with acute lymphoblastic leukemia (ALL) are cured following intensive therapy, the impressive outcomes are not without drawbacks, i.e., short-

Correspondence to: Dr Tamer Hasan Hassan, Pediatric Hematology and Oncology Unit, Zagazig University, Zagazig 44111, Egypt

E-mail: dr.tamerhassan@yahoo.com

Key words: acute childhood leukemia, lymphoblastic leukemia, chemosensitivity, relapse, predictors and long-term morbidities. The identification of prognostic factors and evolution of risk-adapted therapy for children with ALL is one of the success stories in modern clinical oncology. Better indicators means physicians are able to avoid over-treating ALL patients, instead less aggressive protocols are adimistered, with equally promising outcomes (1).

Prognostic models based mostly on pretreatment factors were established and attempted to identify favorable- and poorprognosis patients to assign them to risk-adapted therapies. Although achievement of a complete response (CR) remains the most significant clinical endpoint for survival, criteria for CR are arbitrarily defined. Apart from the questionable numerical cut-off points that characterize $\mathrm{CR}$, remission is not an all-or-none phenomenon. The shorter the time to CR, the better is the long-term outcome in chemotherapy-treated patients. Response during therapy can therefore supplement pretreatment prognostic data (2).

A large number of clinical and biologic prognostic factors were determined during the last 30 years and used to stratify patients to risk-directed therapy. Clinical characteristics included such factors as gender, age, presence of lymphomatous features and white blood count (WBC) at diagnosis. Biological characteristics, including immunophenotype, cytogenetics and lymphoblast morphology, were also used to predict the likelihood of relapse. However, with improved chemotherapy regimens, a number of these traditional prognostic factors have lost clinical significance.

Studies emphasized the significance of response to initial therapy as measured by the reduction in peripheral circulating lymphoblast count, early bone marrow response, and detection of minimal residual disease at the end of induction. The biological response of the malignant lymphoblast population to chemotherapy is crucial to achieving a sustained complete remission and cure (3).

Children receiving cytotoxic chemotherapy for ALL present with significant variations in the incidence and severity of hematologic and non-hematologic side effects, frequently causing delays in scheduled therapy and reduced dose intensity. The variation in side-effect profiles in individual patients remains to be elucidated, apart from rare instances of inherited dysfunction of key drug-metabolizing enzymes involved in cytotoxic drug metabolism, such as thiopurine S-methyltransferase (4). 
It is unknown whether the variation in the individual response of non-malignant hematologic cells to chemotherapy correlates with the risk of relapse or the response of malignant lymphoblasts to chemotherapy in children with ALL (3).

We aimed to improve relapse prediction in children with ALL by evaluating the chemosensitivity of normal and malignant cells early in the course of treatment and determining the relationship between such chemosensitivity and risk of relapse.

\section{Patients and methods}

Patients. A retrospective analysis was conducted on a cohort of 60 children with newly diagnosed ALL. The patients were consecutively enrolled and treated on modified CCG 1991 protocol for standard risk ALL and modified CCG 1961 protocol for high-risk ALL during the period from 2004 to 2009. A total of 40 patients were in complete remission for at least 4 years from the first CR (group I) and 20 patients relapsed during or following treatment (group II). The cut-off point of 4 years was selected as none of the patients in group II had relapse-free survival (RFS) over 4 years and to allow more patients to be included in the study since the modified CCG protocol was assigned in 2004 to be used as a unified Egyptian protocol for treatment of childhood ALL.

Eligibility criteria included patient age between 1 and 15 years; adherence to the modified CCG 1991 protocol for standard risk ALL and modified CCG 1961 protocol for high-risk ALL; remission achieved after a 4-week induction regimen; and appropriate available medical records.

Exclusion criteria included patient age $<1$ year and $>15$ years; failure to achieve remission after a 4-week induction regimen; death during induction; and incomplete or unavailable medical records.

Data collection. A standardized data abstraction form was designed to gather appropriate information from individual patient medical records. The collected data included: i) pretreatment risk factors such as age, clinical presentation, initial total leukocytic count (TLC) and immunophenotyping; ii) the risk group of the patients based on the above-mentioned pretreatment risk factors; iii) complete blood count (CBC) results at diagnosis, during induction and at the end of induction; iv) time to peripheral blood blast clearance (PBBC); and v) RFS in patients of group II. Notably, the absolute neutrophil count (ANC) is the sum of the number of mature neutrophils and band forms expressed in cells per liter. Blast clearance is defined as the time interval from diagnosis to the day where no blast cells were detected in the differential count of peripheral WBCs.

Statistical analysis. Data were entered, checked and analyzed using SPSS version 15. Data were expressed as mean \pm standard deviation (SD) for quantitative variables or the number and percentage for qualitative ones. The Chi-square or Fisher's exact test, t test and correlation coefficient test were utilized when appropriate. Logistic regression was used for prediction of the probability of occurrence of relapse by analysis of various predictor variables.
Table I. Comparison between study group I and II regarding pretreatment risk factors and risk grouping.

\begin{tabular}{|c|c|c|c|c|}
\hline $\begin{array}{l}\text { Pretreatment } \\
\text { risk factor }\end{array}$ & Group I & Group II & Value $^{a}$ & P-value \\
\hline \multicolumn{5}{|l|}{ Age (years) } \\
\hline Mean \pm SD & $6.4 \pm 2.8$ & $7.5 \pm 3.4$ & $\mathrm{t}=1.3$ & 0.19 \\
\hline Range & 3-13 & $2-14$ & & \\
\hline \multicolumn{5}{|l|}{ Gender, n (\%) } \\
\hline Male & $22(55)$ & $11(55)$ & $\chi^{2}=0.00$ & 1.00 \\
\hline Female & $18(45)$ & $9(45)$ & & \\
\hline \multicolumn{5}{|l|}{ CNS, n (\%) } \\
\hline -ve & $39(97.5)$ & $18(90)$ & $\chi^{2}=0.39$ & 0.52 \\
\hline$+v e$ & $1(2.5)$ & $2(10)$ & & \\
\hline \multicolumn{5}{|l|}{ TLC $\left(10^{9} / 1\right)$} \\
\hline Mean \pm SD & $18.98 \pm 28.4$ & $29.9 \pm 38.1$ & $\mathrm{t}=1.25$ & 0.21 \\
\hline Range & 0.4-115 & $0.9-125$ & & \\
\hline \multicolumn{5}{|l|}{ IP } \\
\hline Pre-B-ALL & $33(82.5)$ & $13(65)$ & $\chi^{2}=1.41$ & 0.23 \\
\hline T-ALL & $7(17.5)$ & $7(35)$ & & \\
\hline \multicolumn{5}{|l|}{ Risk } \\
\hline SR & $32(80)$ & $11(55)$ & $\chi^{2}=4.1$ & $0.04^{\mathrm{b}}$ \\
\hline HR & $8(20)$ & $9(45)$ & & \\
\hline
\end{tabular}

aValue of test of significance used. 'bignificant difference. SD, standard deviation; CNS, central nervous system; TLC, total leukocytic count; IP, immunophenotyping; ALL, acute lymphoblastic leukemia; $\mathrm{SR}$, standard risk; HR, high risk.

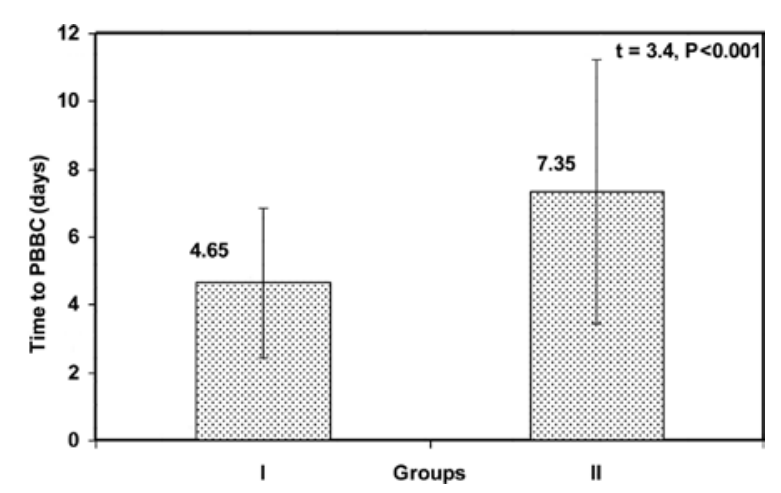

Figure 1. Time to PBBC in study groups I and II. Time to PBBC was significantly shorter in group I than in group II (4.65 \pm 2.2 vs. $7.35 \pm 3.9$ days, respectively; $\mathrm{p}<0.001)$.

\section{Results}

Pretreatment risk factors in the studied groups. No statistical significant difference was noted between groups I and II with regard to pretreatment risk factors including age, gender, central nervous system (CNS) involvement, TLC and immunophenotype ( $\mathrm{p}=0.19,1.00,0.52,0.21$ and 0.23 , respectively). However, a statistically significant difference was found between groups I and II regarding risk grouping $(p=0.04)$, in that $80 \%$ of patients in group I belonged to the standard risk category and $20 \%$ belonged to the high-risk category, while 


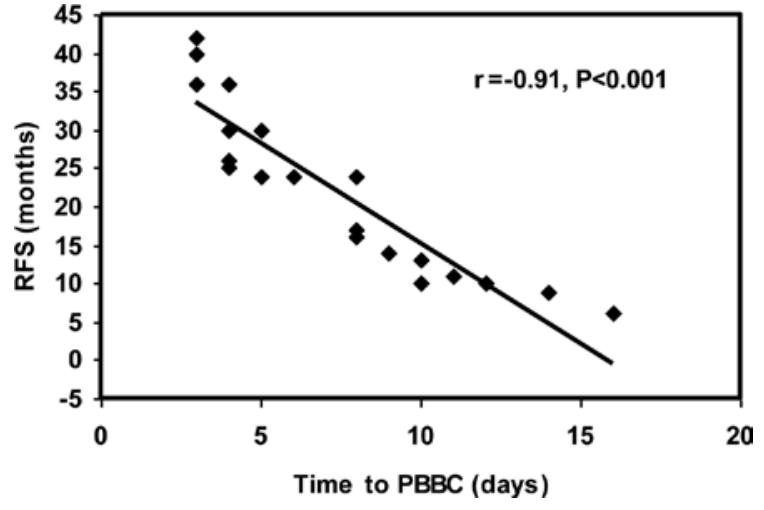

Figure 2. Correlation between time to PBBC and RFS in group II. A highly significant negative correlation was noted between time to PBBC and RFS $(\mathrm{r}=-0.91 ; \mathrm{p}<0.001)$

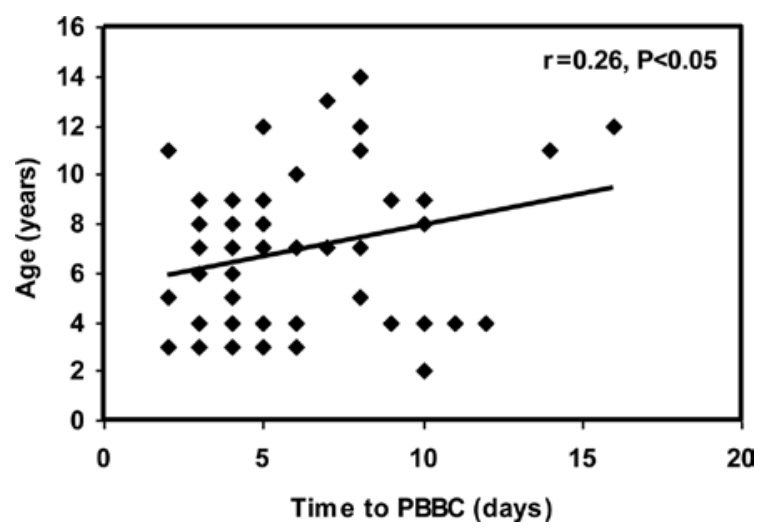

Figure 3. Correlation between time to $\mathrm{PBBC}$ and age at initial diagnosis. A significant positive correlation was noted between time to $\mathrm{PBBC}$ and age at initial diagnosis $(r=0.26 ; \mathrm{p}<0.05)$.

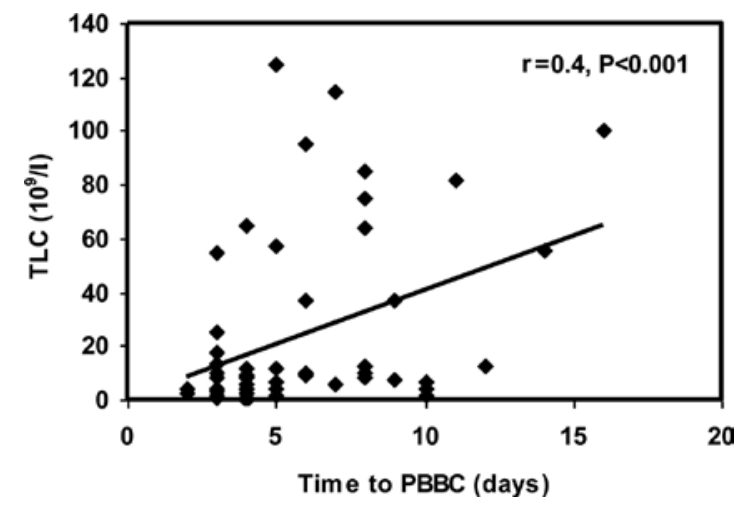

Figure 4. Correlation between time to PBBC and TLC at initial diagnosis. A highly significant positive correlation was noted between time to PBBC and TLC at initial diagnosis $(r=0.40 ; \mathrm{p}<0.001)$.

$55 \%$ of patients in group II were of standard risk and $45 \%$ were of high-risk (Table I).

Time to PBBC in the study groups. Time to PBBC was significantly shorter in group I than in group II (4.65 \pm 2.2 vs. $7.35 \pm 3.9$ days, respectively; $\mathrm{p}<0.001$ ) (Fig. 1).
Table II. Relationship between time to PBBC and other pretreatment risk factors.

\begin{tabular}{lccc}
\hline $\begin{array}{l}\text { Pretreatment } \\
\text { risk factor }\end{array}$ & Mean \pm SD (range) & t-value & P-value \\
\hline $\begin{array}{l}\text { Gender } \\
\text { Male }\end{array}$ & $6.7 \pm 3.4(3-16)$ & 3.50 & $0.001^{\mathrm{a}}$ \\
Female & $4.1 \pm 1.9(2-10)$ & & \\
CNS & & & \\
-ve & $5.3 \pm 2.8(2-14)$ & 2.89 & $0.005^{\mathrm{b}}$ \\
+ ve & $10.3 \pm 4.9(7-16)$ & & \\
IP & & & \\
Pre-B-ALL & $4.9 \pm 2.7(2-14)$ & 3.07 & $0.003^{\mathrm{b}}$ \\
T-ALL & $7.6 \pm 3.4(3-16)$ & & \\
Risk & & & \\
SR & $4.9 \pm 2.4(2-10)$ & 2.67 & $0.009^{\mathrm{b}}$ \\
HR & $7.2 \pm 4(2-16)$ & & \\
\hline
\end{tabular}

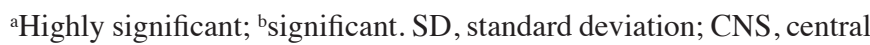
nervous system; IP, immunophenotyping; ALL, acute lymphoblastic leukemia; SR, standard risk; HR, high risk.

Correlation between time to PBBC and RFS in group II. A highly significant negative correlation was noted between time to PBBC and RFS in group II ( $r=-0.91$ and $\mathrm{p}<0.001)$ (Fig. 2).

Correlation between time to PBBC and age and TLC at initial diagnosis. A significant positive correlation was observed between time to PBBC and age at initial diagnosis $(r=0.26$; $\mathrm{p}<0.05)$. In addition, a highly significant positive correlation between time to PBBC and TLC at initial diagnosis ( $\mathrm{r}=0.40$; $\mathrm{p}<0.001$ ) (Figs. 3 and 4) was noted.

Relationship between time to PBBC and other pretreatment risk factors. A significant relationship was found between time to PBBC and males, CNS +ve patients, patients with the T-cell immunophenotype and high-risk patients $(\mathrm{p}=0.001$, $0.005,0.003$ and 0.009 , respectively), with time to $\mathrm{PBBC}$ being longer in the latter patients (Table II).

$C B C$ parameters at the end of induction therapy in the studied groups. End-of-induction TLC and ANC were significantly higher in group I than in group II (6.2 \pm 3.3 vs. $3.4 \pm 3.110^{9} / 1$, respectively, for TLC and $3.3 \pm 1.9$ vs. $1.64 \pm 1.7$, respectively, for ANC). No difference was found between the two groups with regard to hemoglobin level and platelet count at the endof-induction therapy (Table III).

Correlation between end-of-induction ANC and RFS. A non-significant positive correlation was observed between end-of-induction ANC and RFS ( $\mathrm{r}=0.13$; $\mathrm{p}>0.05$ ).

Relationship between ANC and pretreatment risk factors. Low ANC at the end of induction was significantly correlated with the T-cell immunophenotype and high-risk patients $(\mathrm{p}=0.019$, 0.016 , respectively). No differences in end-of-induction ANC and other pretreatment risk factors including age, gender, TLC 
Table III. Comparison between study group I and II with regard to CBC parameters at end of induction therapy.

\begin{tabular}{lccc}
\hline Parameter & Group I & Group II & t-value \\
\hline End-of-induction TLC $\left(10^{9} / 1\right)$ & & & 3.19 \\
Mean \pm SD & $6.2 \pm 3.3$ & $3.4 \pm 3.1$ & $0.001^{\mathrm{a}}$ \\
$\quad$ Range & $0.7-12.5$ & $0.44-10.3$ & 3.35 \\
End-of-induction ANC $\left(10^{9} / \mathrm{l}\right)$ & & & \\
Mean \pm SD & $3.3 \pm 1.9$ & $1.64 \pm 1.7$ & $0.001^{\mathrm{a}}$ \\
Range & $0.28-6.8$ & $0.11-5.2$ & 1.68 \\
End-of-induction PLT count $\left(10^{9} / 1\right)$ & & $120.7 \pm 60.4$ & 0.090 \\
Mean \pm SD & $146.0 \pm 50.3$ & $44-285$ & 1.84 \\
Range & $81-265$ & & \\
End-of-induction Hb $(\mathrm{g} / \mathrm{dl})$ & & $9.9 \pm 1.1$ & 0.060 \\
Mean \pm SD & $10.4 \pm 1.0$ & $7.9-11.8$ & \\
Range & $7.8-11.7$ & & \\
\hline
\end{tabular}

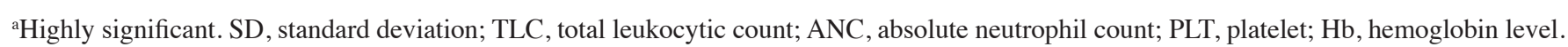

Table IV. Relationship between ANC and pretreatment risk factors.

\begin{tabular}{|c|c|c|c|c|}
\hline \multirow[b]{2}{*}{$\begin{array}{l}\text { Pretreatment } \\
\text { risk factor }\end{array}$} & \multicolumn{2}{|c|}{ ANC } & \multirow[b]{2}{*}{ Value $^{\mathrm{a}}$} & \multirow[b]{2}{*}{ P-value } \\
\hline & $\begin{array}{l}\text { Low } \\
n=14\end{array}$ & $\begin{array}{c}\text { Average } \\
n=46\end{array}$ & & \\
\hline \multicolumn{5}{|l|}{ Age (years) } \\
\hline Mean \pm SD & $7.8 \pm 3.7$ & $6.5 \pm 2.75$ & $\mathrm{t}=1.43$ & 0.150 \\
\hline \multicolumn{5}{|l|}{ Gender, n (\%) } \\
\hline $\begin{array}{l}\text { Male } \\
\text { Female }\end{array}$ & $\begin{array}{l}8(57.1) \\
6(42.9)\end{array}$ & $\begin{array}{l}25(54.3) \\
21(45.7)\end{array}$ & $\chi^{2}=0.03$ & 0.850 \\
\hline \multicolumn{5}{|l|}{ CNS, n (\%) } \\
\hline $\begin{array}{l}\text {-ve } \\
+\mathrm{ve}\end{array}$ & $\begin{array}{r}12(85.7) \\
2(14.3)\end{array}$ & $\begin{array}{c}45(97.8) \\
1(2.2)\end{array}$ & $\chi^{2}=1.26$ & 0.260 \\
\hline \multicolumn{5}{|l|}{ TLC (10/1) } \\
\hline $\begin{array}{l}\mathrm{IP}, \mathrm{n}(\%) \\
\text { Pre-B-ALL } \\
\text { T-ALL }\end{array}$ & $\begin{array}{l}7(50) \\
7(50)\end{array}$ & $\begin{array}{r}39(84.8) \\
7(15.2)\end{array}$ & $\chi^{2}=5.44$ & $0.019^{\mathrm{b}}$ \\
\hline $\begin{array}{l}\text { Risk, n (\%) } \\
\text { SR } \\
\text { HR }\end{array}$ & $\begin{array}{l}6(42.9) \\
8(57.1)\end{array}$ & $\begin{array}{r}37(80.4) \\
9(19.6)\end{array}$ & $\chi^{2}=5.73$ & $0.016^{\mathrm{b}}$ \\
\hline
\end{tabular}

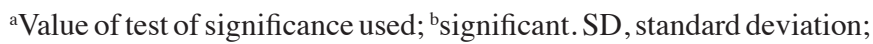
CNS, central nervous system; TLC, total leukocytic count; IP, immunophenotyping; ALL, acute lymphoblastic leukemia; SR, standard risk; HR, high risk.

and CNS involvement were noted $(\mathrm{p}=0.15,0.85,0.26$ and 0.13 , respectively) (Table IV).

Correlation between time to $P B B C$ and $C B C$ parameters at end of induction. A non-significant negative correlation was observed between time to PBBC and any of the $\mathrm{CBC}$ parameters at end-of-induction therapy $(\mathrm{p}>0.05)$ (Table V).
Table V. Correlation between time to PBBC and CBC parameters at end-of-induction therapy.

\begin{tabular}{lccc}
\hline $\begin{array}{l}\text { CBC parameters } \\
\text { at end of induction }\end{array}$ & $\mathrm{r}$ & P-value & Significance \\
\hline TLC & -0.12 & $>0.05$ & $\mathrm{NS}$ \\
ANC & -0.16 & $>0.05$ & $\mathrm{NS}$ \\
PLT & -0.07 & $>0.05$ & $\mathrm{NS}$ \\
$\mathrm{Hb}$ & -0.11 & $>0.05$ & $\mathrm{NS}$
\end{tabular}

NS, not significant. TLC, total leukocytic count; ANC, absolute neutrophil count; PLT, platelet count; $\mathrm{Hb}$, hemoglobin level.

Predictors of relapse in our study. In univariate analyses, our results showed that high-risk patients, longer time to $\mathrm{PBBC}$, low end-of-induction TLC and low end-of-induction ANC were significantly associated with increased risk of relapse ( $\mathrm{p}=0.04,0.001,0.001$ and 0.001, respectively). By applying logistic regression for prediction of the probability of occurrence of relapse by analysis of these predictors, we found that the time to PBBC was the only predictor for occurrence of relapse $(\beta \pm$ standard error $=0.051 \pm 0.018 ; \mathrm{p}<0.001)$. However, risk categorization, end-of-induction TLC and end-of-induction ANC were found not to add significance to this model.

\section{Discussion}

Approximately $80 \%$ of children and adolescents with ALL can be cured. To reduce the rate of relapses, but also to limit treatment toxicity, risk-adapted treatment has been attempted following the identification of the most specific prognostic factors. In addition to clinical factors such as age and WBC, or leukemia cell factors such as immunophenotype and cytogenetics, the in vivo response to therapy has evolved as the most significant predictor for relapse (1). 
The rapidity with which leukemia cells are eliminated following onset of treatment is associated with outcome, as is the level of residual disease at the end of induction therapy. Since treatment response is affected by the drug sensitivity of leukemic cells and host pharmacodynamics and pharmacogenomics (5), this measure has strong prognostic significance. In the present study, the time to PBBC was significantly shorter in group I than in group II $(4.65 \pm 2.2$ vs. $7.35 \pm 3.9$ days, respectively; $\mathrm{p}<0.001)$. This is in agreement with previous studies conducted by Rautonen et al (6) and Gajjar et al (7) who concluded that persistence of circulating blasts following 1 week of multiagent chemotherapy confers a poor prognosis in childhood ALL. Similarly, Laughton et al (3) studied the level of chemoresponsiveness of ALL blasts in vivo and reported that a time greater than a median of 4 days to the disappearance of peripheral lymphoblasts was also associated with an increased risk of relapse.

Our results showed that there was a highly significant negative correlation between time to PBBC and RFS ( $r=-0.91$ and $\mathrm{p}<0.001$ ), and a shorter time to PBBC was associated with a longer RFS. Gajjar et al (7) found that patients with a more rapid response in terms of PBBC had an RFS of 64 vs. 39\% for slower responders. The same finding was reported in a Pediatric Oncology Group study conducted by Griffin et al (8). A study published by investigators from Mayo Clinic and Northwestern University showed that, as in childhood ALL, the time to clearance of circulating blasts from peripheral blood also serves as an independent prognostic marker of RFS and overall survival (OS) for AML (9).

In the present study, there was a highly significant positive correlation between time to PBBC and TLC at initial diagnosis $(r=0.40$ and $p<0.001)$ as well as a significant positive correlation between time to PBBC and age at initial diagnosis $(r=0.26$ and $p<0.05)$. Regarding other pretreatment risk factors, there was a significant relationship between time to $\mathrm{PBBC}$ and males, CNS +ve patients, patients with a T-cell immunophenotype and high-risk patients $(\mathrm{p}=0.001,0.005$, 0.003 and 0.009 , respectively) with time to PBBC being longer in the latter patients. Gajjar et al (7) found that patients with a persistence of circulating blasts after 1 week of multiagent chemotherapy were more likely to have adverse presenting features, such as increased leukocyte count, mediastinal mass, CNS leukemia, T-cell phenotype and lack of CDl0 expression, while no relationship was found with patient age. In addition, Manabe et al (10) reported that $25.6 \%$ of T-ALL patients were classified in the Day8NoBlasts, whereas $34.0 \%$ of B-ALL patients belonged to the Day8NoBlasts group.

Our study showed that end-of-induction TLC and ANC were significantly higher in group I than in group II patients (6.2 \pm 3.3 vs., $3.4 \pm 3.110^{\%} / 1$, respectively, for TLC and $3.3 \pm 1.9$ vs. $1.64 \pm 1.7$, respectively, for ANC). Our results indicate that a more rapid recovery of end-of-induction TLC and ANC correlated with a reduced risk of relapse. Additional measures of myelosuppression at the end of induction, including hemoglobin level and platelet count, did not show any difference between the studied groups. Our results corroborate those reported by Laughton et al (3) who found a significant association between risk of relapse and both low TLC and ANC at the end of induction. These authors found that neither hemoglobin level nor platelet count at the end of induction was associated with increased risk of relapse. De Angulo and colleagues (11), in their study on 171 de novo ALL and AML patients $\leq 21$ years of age, found that the end-of-induction platelet count was not predictive of relapse in ALL patients. However, these authors found that a platelet count of $\geq 100,000$ on Day 28 was able to predict favorable OS for patients with AML. Platelet recovery has also been reported to be a prognostic marker in older adults with ALL (12).

The mechanism whereby a more rapid recovery of end-ofinduction ANC correlates with a reduced risk of later relapse has yet to be elucidated. One possibility is that end-of-induction ANC is a general marker of the ability of a patient to exhibit normal hematologic cell recovery following chemotherapy.

Although a highly significant negative correlation was found between time to PBBC and RFS, we found a nonsignificant positive correlation between end-of-induction ANC and RFS. Contrary to our data, Laughton et al (3) found that end-of-induction ANC was highly significantly associated with RFS $(\mathrm{p}<0.0001)$.

In the present study, low ANC at the end of induction was significantly associated with T-cell immunophenotype and high-risk patients. No differences in end-of-induction ANC and other pretreatment risk factors including age, gender, TLC and CNS affection were noted. Laughton et al (3) found that there were no differences in patient characteristics at initial diagnosis, such as age or TLC, for patients with a low ANC at end-of-induction therapy.

We investigated the correlation between time to PBBC, which reflects the chemosensitivity of malignant lymphoblasts, and the CBC parameters at end of induction, which reflect the chemosensitivity of normal hematopoietic cells. We found that these factors were not significantly correlated, confirming that they behave independently in response to chemotherapy. Laughton et al (3) reported that the early peripheral blast response was not associated with the end-ofinduction ANC.

In conclusion, we showed that time to PBBC as a measure of chemosensitivity of malignant cells, and end-of-induction TLC and ANC as a measure of chemosensitivity of normal hematopoietic cells in ALL patients can improve prediction of relapse, allowing for the modification of chemotherapy intensity at an earlier time point during the course of therapy. A wider prospective, randomized, controlled trial is required to confirm our results.

\section{References}

1. Schrappe M: Prognostic factors in childhood acute lymphoblastic leukemia. Indian J Pediatr 7: 817-824, 2003.

2. Faderl S and Estrov Z: Hematopoietic recovery following induction therapy of acute leukemias: prognostic implications and a new look at the definition of remission. Leuk Lymphoma 45: 67-71, 2004.

3. Laughton SJ, Ashton LJ, Kwan E, Norris MD, Haber M and Marshall GM: Early responses to chemotherapy of normal and malignant hematologic cells are prognostic in children with acute lymphoblastic leukemia. J Clin Oncol 23: 2264-2271, 2005.

4. McLeod HL, Krynetski EY, Relling MV and Evans WE: Genetic polymorphism of thiopurine methyltransferase and its clinical relevance for childhood acute lymphoblastic leukemia. Leukemia 14: 567-572, 2000.

5. Relling MV and Dervieux T: Pharmacogenetics and cancer therapy. Nat Rev Cancer 1: 99-108, 2001. 
6. Rautonen J, Hovi L and Siimes MA: Slow disappearance of peripheral blast cells: an independent risk factor indicating poor prognosis in children with acute lymphoblastic leukemia. Blood 71: 989-991, 1998.

7. Gajjar A, Ribeiro R, Hancock ML, Rivera GK, Mahmoud H, Sandlund JT, Crist WM and Pui CH: Persistence of circulating blasts after 1 week of multiagent chemotherapy confers a poor prognosis in childhood acute lymphoblastic leukemia. Blood 86 : $1292-1295,1995$

8. Griffin TC, Shuster JJ, Buchanan GR, Murphy SB, Camitta BM and Amylon MD: Slow disappearance of peripheral blood blasts is an adverse prognostic factor in childhood T cell acute lymphoblastic leukemia: a Pediatric Oncology Group study. Leukemia 14: 792-795, 2000.

9. Elliott MA, Litzow MR, Letendre LL, Wolf RC, Hanson QA, Tefferi A and Tallman MS: Early peripheral blood blast clearance during induction chemotherapy for acute myeloid leukemia predicts superior relapse-free survival. Blood 110: 4172-4174, 2007.
10. Manabe A, Ohara A, Hasegawa D, Koh K, Saito T, Kiyokawa N, Kikuchi A, Takahashi H, Ikuta K, Hayashi Y, Hanada R and Tsuchida M: Significance of the complete clearance of peripheral blasts after 7 days of prednisolone treatment in children with acute lymphoblastic leukemia: the Tokyo Children's Cancer Study Group Study L99-15. Haematologica 93: 1155-1160, 2008.

11. De Angulo G, Yuen C, Palla SL, Anderson PM and Zweidler-McKay PA: Absolute lymphocyte count is a novel prognostic indicator in ALL and AML: implications for risk stratification and future studies. Cancer 112: 407-415, 2008.

12. Faderl S, Thall PF, Kantarjian HM and Estrov Z: Time to platelet recovery predicts outcome of patients with de novo acute lymphoblastic leukemia who have achieved a complete remission. Br J Haematol 117: 869-874, 2002. 